\title{
Efficient Target Geolocation by Highly Uncertain Small Air Vehicles
}

\author{
Ben Grocholsky, Michael Dille, and Stephen Nuske \\ Robotics Institute, Carnegie Mellon University
}

\begin{abstract}
Geolocation of a ground object or target of interest from live video is a common task required of small and micro unmanned aerial vehicles (SUAVs and MAVs) in surveillance and rescue applications. However, such vehicles commonly carry low-cost and light-weight sensors providing poor bandwidth and accuracy whose contribution to observations is nonlinear, resulting in poor geolocation performance by standard techniques. This paper proposes the application of an efficient over-parameterized state representation to the problem of geolocation that is able to handle large, time-varying, and non-Gaussian sensor error to produce better geolocation estimates than typical approaches and which provides computing and communication benefits in applications such as predictive control and distributed collaboration. We evaluate our filter on real flight data, demonstrating its ability to efficiently produce a solution with tight confidence bounds given highly uncertain data.
\end{abstract}

\section{INTRODUCTION}

Small unmanned and micro air vehicles (SUAVs and MAVs) are increasingly popular in a variety of remote sensing applications such as surveillance, search and rescue, and aerial surveys. Such smaller vehicles are often preferred over larger aircraft because their lower cost permits their use in lower-budget and higher-risk applications, their smaller size and weight often enables human-portability, and their simplicity and lack of infrastructure requirements typically allows rapid launches by fewer minimally trained operators in the field, nearer to where they are required.

These benefits bring downsides, however, as substantial sacrifices in available on-board computing and sensing must be made to meet cost, size, and weight constraints. These are often limited to low-bandwidth, consumer-grade GPS, magnetometer, and inertial measurement devices for vehicle state sensing, and relatively low-resolution cameras with minimal processing prior to down-linking for external sensing. At the same time, the light weight and simple design of such vehicles allows for minimal rejection of disturbances such as wind, limiting control authority and worsening estimation accuracy.

A common task required of nearly all applications is the geolocation of objects on the ground - mapping a pixel location in the camera's view into world coordinates - for display on a map to an operator, to direct other agents to needed locations, or for later correlation with other data. This is greatly complicated by the considerable inaccuracies in vehicle state, leading to highly inaccurate geolocation measurements. With appropriate representation even such

Pittsburgh, Pennsylvania, USA, 15213 (Email:grocholsky@ri.cmu.edu) large uncertainties may be handled naturally to provide useful geolocation estimates given sufficient observations.

This paper presents uncertainty modeling and observationfusion approaches that produce considerable improvement in geolocation accuracy compared to conventional parametric strategies in the presence of highly nonlinear observation functions and large observer orientation error. The filter uses an over-parameterized state vector that can produce a far closer approximation of the true uncertainty. The result is accurate solutions with tight confidence bounds despite highly uncertain observations.

Their efficacy is then shown in field experiments using a commercial hand-launched UAV. Extensions to visuallytracked moving ground objects are then discussed, concluding with ongoing avenues of further exploration.

\section{RElATED WORK}

Mapping the image location of a point of interest in a UAV's camera view can be performed in a variety of ways. The simplest is to use the vehicle's pose provided by onboard sensors to project the image coordinate as a ray in world coordinates [1] that may then be intersected with a terrain model [2] to produce a location on the ground. Another strategy is registration of live video to previously acquired satellite imagery [3], which provides a direct measurement of a viewable object's world location. Either of these may be combined with simultaneous visual UAV pose refinement techniques such as Extended Kalman Filter (EKF) visual landmark tracking [4], [5], structure from motion [6], and homography-based pose refinement [7] for additional observation accuracy. However, terrain matching techniques assume the availability of recent prior imagery and are vulnerable to changes since this imagery was acquired. Visual odometry techniques typically rely on the ability to persistently track features across many frames despite the potential for occluding and homogeneous terrain. Both classes tend to assume higher quality video than is likely to be provided by a small UAV and present a substantial computational burden. Thus, the more common strategy of ray intersection with terrain is chosen.

A series of observations represented as rays may be combined by finding their best intersection [6] or by batchcombining their intersections with the ground to remove symmetric biases throughout an orbit of a ground object [1]. Various formulations of the Kalman filter have also been applied [8], [9], [10] with varying success, but such attempts fall short due to either using arbitrary observation uncertainties or by relying on linearization of the ray 
intersection function (which will be shown to be a poor approximation given realistic UAV state error), resulting in filter inconsistency.

The Unscented Transform and the resulting Unscented Kalman filter [11] provide some improvement but ultimately assume Gaussian-distributed uncertainties, which will also be shown to be far from true. Particle filters [12] provide another means to approximate the oddly-shaped distributions resulting from multiple geolocation observations but are difficult to tune in this application given strong sensitivity to the accuracy of the target process model (as slow but discontinuous drift in observation error may result in overconfident clustering at an incorrect location) and the need for delicate outlier rejection (as inlier observations may themselves be highly erroneous). This leads in practice to requiring a very large number of particles for accurate geolocation and to avoid particle depletion, resulting in poor performance on embedded hardware or when used for predictive planning requiring frequent likelihood evaluation. Given the dominant heading uncertainty observed in the literature [6], [8], [13], a polar representation may be more appropriate, and inspiration may be drawn from efforts at range-only localization [14].

The authors previously presented [15] a sampling-based evidence grid filter that makes no assumptions about the form of observation or a-posteriori uncertainty distributions and provides accurate estimates in the presence of large, nonGaussian, and time-varying errors in vehicle state, particularly heading. However, it is not easily extended to moving targets, it provides only a very conservative measure of estimate confidence, and while it runs in real-time for grid sizes of several meters and sampling along several state axes, increasing grid resolution and sampling along many additional axes could result in impractical runtimes. This work explores an efficient parametric representation of target location uncertainty and provides a comparison to this and simpler existing methods.

\section{SMAll UAV TARget Geolocation}

\section{A. Geometric Modeling}

The basic geolocation task of mapping a camera pixel location to world coordinates is framed as ray intersection with a terrain model. Assuming the typical pinhole camera model, this ray is given by

$$
\hat{\mathbf{v}}_{\mathrm{obj}}^{\mathrm{cam}} \equiv \mathbf{M}^{-1}\left[\begin{array}{c}
\mathbf{x}_{\mathrm{obj}}^{\mathrm{img}} \\
1
\end{array}\right],
$$

when expressed in homogeneous coordinates, so that an object's world location must satisfy

$$
\mathbf{x}_{\mathrm{obj}}^{\text {world }}=\mathbf{T}_{\mathrm{UAV}}^{\text {world }} \mathbf{p}_{\mathrm{cam}}^{\mathrm{UAV}}+d \mathbf{R}_{\mathrm{UAV}}^{\text {world }} \mathbf{R}_{\mathrm{cam}}^{\mathrm{UAV}} \hat{\mathbf{v}}_{\mathrm{obj}}^{\mathrm{cam}}, d \in \mathbb{R},
$$

where $d$ is the distance to the object along the ray, $\mathbf{T}_{i}^{j}$ is the pose transformation taking coordinates in frame $i$ to $j$, $\mathbf{R}_{i}^{j}$ is the corresponding rotation matrix, $\mathbf{p}_{i}^{j}$ is the position of body $i$ in the coordinate system of $j, \mathbf{M}$ is the $3 \times 3$ camera calibration matrix, and $\mathrm{x}_{\mathrm{obj}}^{\mathrm{img}}$ are the pixel coordinates of the visible target. For simplicity, we here assume locally flat terrain so that

$$
d=\left(z_{\text {terrain }}-z_{\mathbf{p}_{\text {cam }}^{\text {world }}}\right) / z_{\hat{\mathbf{v}}_{\text {obj }}^{\text {world }}} .
$$

The process of determining $d$ may be represented by a function $f_{\text {obs }}\left(\mathbf{T}_{\text {world }}^{\mathrm{UAV}}, \mathbf{T}_{\mathrm{UAV}}^{\mathrm{cam}}, \mathbf{x}_{\mathrm{obj}}^{\mathrm{mg}}\right)$ providing an observation of the target's world location $\mathbf{x}_{\mathrm{obj}}^{\text {world }}$ given UAV state and calibrated camera pose.

\section{B. Nature of Uncertainty}

A number of error sources contribute to inaccuracy in geolocation, in the form of inaccuracy in any of the preceding pose transformations. Relative camera pose $\mathbf{p}_{\mathrm{cam}}^{\mathrm{UAV}}$ and $\mathbf{R}_{\mathrm{cam}}^{\mathrm{UAV}}$ can be measured to fairly minute levels, standard calibration methods can be applied to determine $\mathbf{M}$ to high accuracy, and GPS error providing $\mathbf{p}_{\mathrm{UAV}}^{\text {world }}$ contributes additively, typically at the level of up to several meters. UAV orientation $\mathbf{R}_{\mathrm{UAV}}^{\text {world }}$, however, has an immense effect, as $d$ is generally a large value on the order of several hundred meters. Heading error is typically the largest as no absolute reference other than Earth's weak magnetic field is available. This has the effect of making linearization of $f_{o b s}$ for Jacobian transformation of state uncertainty to observation uncertainty a very poor approximation, as shown in Figure 1. Additionally, this shows that constraining observation uncertainty to a Gaussian distribution produces an uninformative, over-conservative result. Worse, empirical data from live UAV tests excerpted in Figure 2 have shown that heading error provided by stock autopilots may be large, time-varying, and non-Gaussian in distribution. Meanwhile, Figure 3 shows that range error is proportionately smaller, more tightly clustered, and easily bounded if not entirely Gaussian. This motivates a conservative polar representation for observations with bounded uncertainty that are then intersected to produce a filtered result.

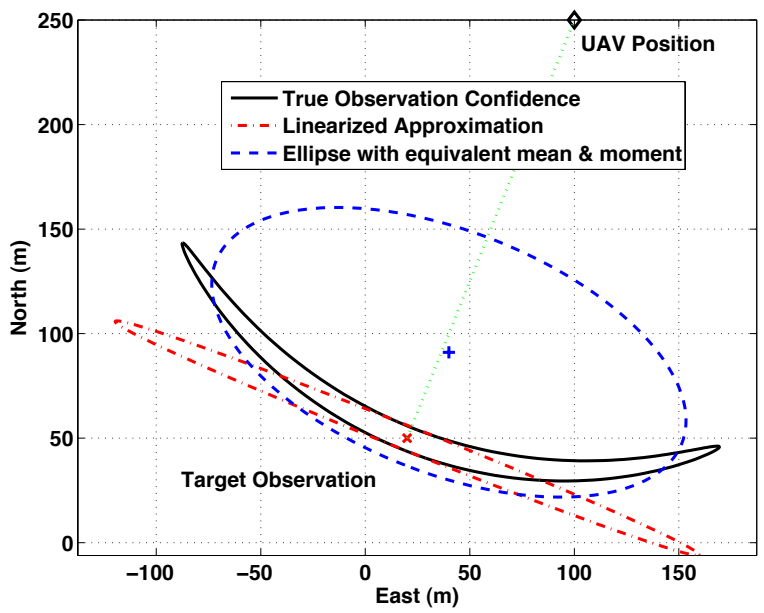

Fig. 1: UAV observed target location uncertainty

\section{EFFicient Nonlinear State Estimation}

Our approach reformulates a class of nonlinear problems such that they can be handled by established linear methods. 

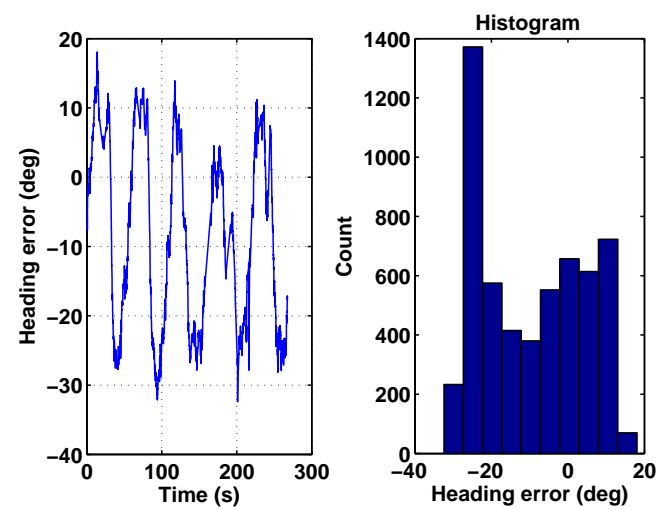

Fig. 2: Observed UAV target heading error
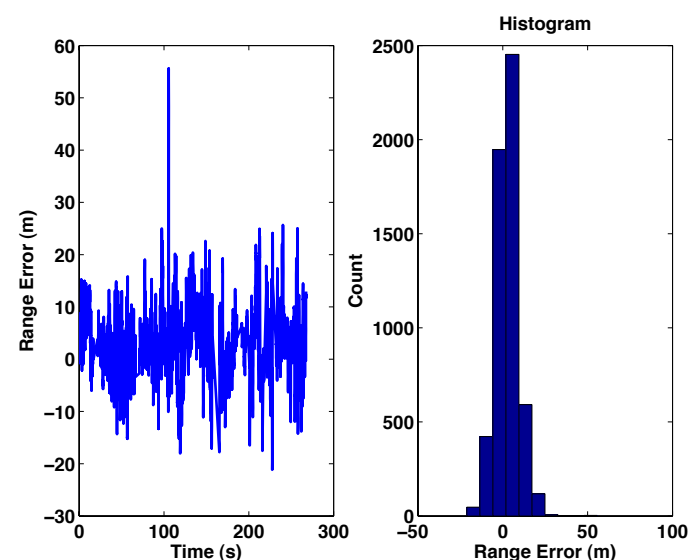

Fig. 3: Observed UAV target range error

\section{A. Recursive Bounded-Error Estimation}

Schweppe [16] proposed a recursive filtering method for the case where the uncertain state, process disturbances and observation noise are represented by bounded ellipsoidal sets. The estimate $\hat{\mathbf{x}}$ of the true state $\mathbf{x}$ is described by

$$
\tilde{\boldsymbol{\Sigma}}=\left\{\mathbf{x}:[\mathbf{x}-\hat{\mathbf{x}}]^{T} \boldsymbol{\Sigma}^{-1}[\mathbf{x}-\hat{\mathbf{x}}] \leq 1\right\},
$$

where $\boldsymbol{\Sigma}^{-1}$ is a positive semi-definite moment matrix analogous to covariance for Gaussian error models. Dynamics and measurements are represented by linear stochastic process and observation models

$$
\begin{gathered}
\mathbf{x}(k+1 \mid k)=\mathbf{\Phi}\left(t_{k+1}-t_{k}\right) \mathbf{x}(k \mid k)+\mathbf{G u}, \\
\mathbf{z}(k)=\mathbf{H x}(k)+\mathbf{v} .
\end{gathered}
$$

With process and observation noise

$$
\left\{\mathbf{u}: \mathbf{u}^{T} \mathbf{Q}^{-1} \mathbf{u} \leq 1\right\} \text { and }\left\{\mathbf{v}: \mathbf{v}^{T} \mathbf{R}^{-1} \mathbf{v} \leq 1\right\} .
$$

The estimation method involves two stages described by Equations 8 through 12 that are similar to the well known Kalman filter for Gaussian uncertainty models.

\section{Predict:}

$$
\begin{gathered}
\hat{\mathbf{x}}(k+1 \mid k)=\mathbf{\Phi}\left(t_{k+1}-t_{k}\right) \hat{\mathbf{x}}(k \mid k) \\
\boldsymbol{\Sigma}(k+1 \mid k)=\alpha_{k}\left[\boldsymbol{\Phi}\left(t_{k+1}-t_{k}\right) \boldsymbol{\Sigma}(k \mid k) \boldsymbol{\Phi}^{T}\left(t_{k+1}-t_{k}\right)+\mathbf{Q}\right]
\end{gathered}
$$

where $\alpha_{k} \in[1,2]$.
Update:

$$
\begin{aligned}
& \hat{\mathbf{x}}(k \mid k)=\hat{\mathbf{x}}(k \mid k-1)+\rho_{k}\left[\boldsymbol{\Sigma}^{-1}(k \mid k-1)\right. \\
& \left.+\rho_{k} \mathbf{H}^{T} \mathbf{R}^{-1} \mathbf{H}\right]^{-1} \mathbf{H}^{T} \mathbf{R}^{-1}\left[\mathbf{z}_{k}\right. \\
& -\mathbf{H} \hat{\mathbf{x}}(k \mid k-1)] \\
& \boldsymbol{\Sigma}(k \mid k)=\eta_{k}\left[\boldsymbol{\Sigma}^{-1}(k \mid k-1)+\rho_{k} \mathbf{H}^{T} \mathbf{R}^{-1} \mathbf{H}\right]^{-1} \\
& \eta_{k}=1+\rho_{k}-\left[\mathbf{z}_{k}-\mathbf{H} \hat{\mathbf{x}}(k \mid k-1)\right]^{T}\left[\rho_{k}^{-1} \mathbf{R}\right. \\
& \left.+\mathbf{H} \boldsymbol{\Sigma}(k \mid k-1) \mathbf{H}^{T}\right]^{-1}\left[\mathbf{z}_{k}-\mathbf{H} \hat{\mathbf{x}}(k \mid k-1)\right]
\end{aligned}
$$

for non-negative $\rho_{k}$.

Conceptually these equations use set summation and intersection to perform estimation. The key algebraic difference to the Kalman filter is two scalar parameters $\alpha_{k}$ and $\rho_{k}$ that adjust the set volume produced by sum and intersection operations. Known guaranteed constant values $\left(\alpha_{k}=2\right.$ and $\left.\rho_{k}=0.5\right)$ prove too conservative in practice. The implementation used in this paper performs a scalar convex optimization at each time step to find the bounded estimate with minimum set volume. Observations that do not intersect the predicted estimate are detected and rejected as outliers.

\section{B. Over-Parameterized State Representation}

Application of linear bounded ellipsoidal estimation methods to non-linear problems avoiding coarse linearization approximation was demonstrated by Hanebeck [17]. The approach relies on finding a nonlinear embedding that maps the system state into an higher-dimensional state space where the measurement equations become linear.

The embedding considered throughout this paper for twodimensional target geo-location problems is simply

$$
\mathbf{x}=\left[\begin{array}{l}
x \\
y
\end{array}\right] \quad \rightarrow \quad \mathbf{x}^{\star}=\left[\begin{array}{c}
x \\
y \\
x^{2}+y^{2}
\end{array}\right]
$$

a special case of the embedding used previously by the authors in [18] for range-only SLAM.

Linear estimation in this three dimensional space is able to express a diverse range of non-linear, non-convex and nonsimply connected sets with applicability to non-linear sensing problems. This point is illustrated by an example presented in Figures 4 and 5. Figure 4 shows a rank 2 hyper-ellipse that intersects the embedding manifold at two locations resulting in a bi-modal crescent shaped estimate. Figure 5 shows iterations of an isotropic diffusion process applied to this initial condition. The bi-modal disconnected sets transition to become an annulus and eventually uni-modal.

\section{Computing Estimate Sets and Confidence Metrics}

A challenge in using over-parameterized state representations is determining the estimate sets in the lower dimensional state space. This step involves projecting the intersection of the higher-dimensional ellipse with the embedding manifold down into the original state space. In general this poses a daunting computational challenge. However, for the space $\left\{x, y, x^{2}+y^{2}\right\}^{T}$, the implicit description of the estimate level set Equation 4 is fourth order in $\{x, y\}$ and 


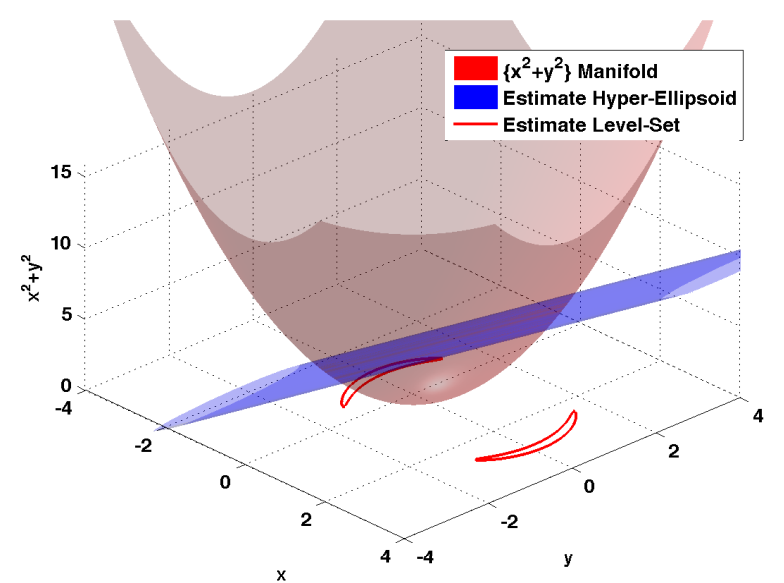

Fig. 4: Example ellipsoidal set visualized in the 3D overparameterized $\mathrm{x}^{\star}$ space and projected into the $2 \mathrm{D}$ state space

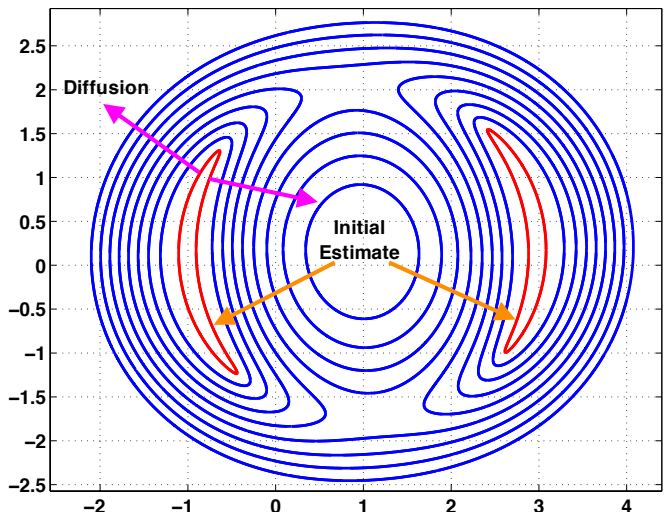

Fig. 5: Example motion model - Isotropic diffusion demonstrates the ability of the 3D over-parameterized ellipsoidal representation to express a rich range of $2 \mathrm{D}$ estimate sets.

possesses a known explicit analytic solution. Thus, estimate level sets and metrics can be directly computed.

Two approximations with minimal to low computation are considered and displayed in Section VI. The first is a guaranteed conservative ellipsoidal bound obtained by simply projecting the over-parameterized ellipse into the original linear state space. This approximation is very fast to compute (linear sub-space of $\boldsymbol{\Sigma}^{\star-1}$ ) but may yield unbounded or highly conservative results. A tighter bound is obtained by computing the smallest axis-aligned bounding box containing all components of the nonlinear estimate set.

\section{Application to Small UAV Target Tracking}

\section{A. Polar Approximation of Observation Uncertainty}

Section III-B highlighted that the observation uncertainty for small UAVs is well characterized in polar coordinates local to the UAV position. This section describes the formulation of a tight approximation to observation uncertainty in the over-parameterized state space.

Equation 2 is used to determine target range and bearing as a function of the uncertain image measurement $\mathbf{z}$, UAV state $\mathbf{x}_{U A V}$ and camera calibration $\mathbf{x}_{C a m}$

$$
\left[\begin{array}{c}
r \\
\beta
\end{array}\right]=f_{r \beta}\left(\mathbf{z}, \mathbf{x}_{U A V}, \mathbf{x}_{C a m}\right) .
$$

The range and bearing uncertainty is approximated by projecting ellipsoidal measurement, state and calibration uncertainty into the local polar coordinate system

$$
\begin{aligned}
\boldsymbol{\Sigma}_{r \beta}= & {\left[\begin{array}{cc}
\sigma_{r}^{2} & \sigma_{r} \sigma_{\beta} \\
\sigma_{r} \sigma_{\beta} & \sigma_{\beta}^{2}
\end{array}\right]=\nabla_{\mathbf{z}} f_{r \beta} \mathbf{R} \nabla_{\mathbf{z}} f_{r \beta}^{T} } \\
& +\nabla_{\mathbf{x}_{\mathrm{UAV}}} f_{r \beta} \boldsymbol{\Sigma}_{U A V} \nabla_{\mathbf{x}_{\mathrm{UAV}}} f_{r \beta}^{T} \\
& +\nabla_{\mathbf{x}_{\mathrm{Cam}}} f_{r \beta} \boldsymbol{\Sigma}_{C a m} \nabla_{\mathbf{x}_{\mathrm{Cam}}} f_{r \beta}^{T} .
\end{aligned}
$$

From this, an approximate observation in the overparameterized space is constructed by combining annular and cylindrical sets that capture the range (Equation 17) and bearing bounds (Equation 18) as illustrated in Figure 6.

Range Bound:

$$
\begin{aligned}
\mathbf{z}_{\text {range }}^{\star} & =r^{2}-\left(x_{\text {uav }}^{2}+y_{\text {uav }}^{2}-\sigma_{r}^{2}\right) \\
\mathbf{H}_{\text {range }}^{\star} & =\left[-2 x_{\text {uav }}-2 x_{\text {uav }} 1\right] \\
\mathbf{R}_{\text {range }}^{\star} & =\left(2 r \sigma_{r}\right)^{2}
\end{aligned}
$$

Bearing Bound:

$$
\begin{aligned}
\mathbf{z}_{\text {circle }} & =\left[\begin{array}{l}
x_{u a v} \\
y_{u a v}
\end{array}\right]+r\left[\begin{array}{c}
\cos \beta \\
\sin \beta
\end{array}\right] e^{-\sigma_{\beta}^{2} / 2} \\
\mathbf{R}_{\text {circle }} & =\left(2 r \sin \left(\sigma_{\beta} / 2\right)\right)^{2} \mathbf{I}_{2 x 2}
\end{aligned}
$$

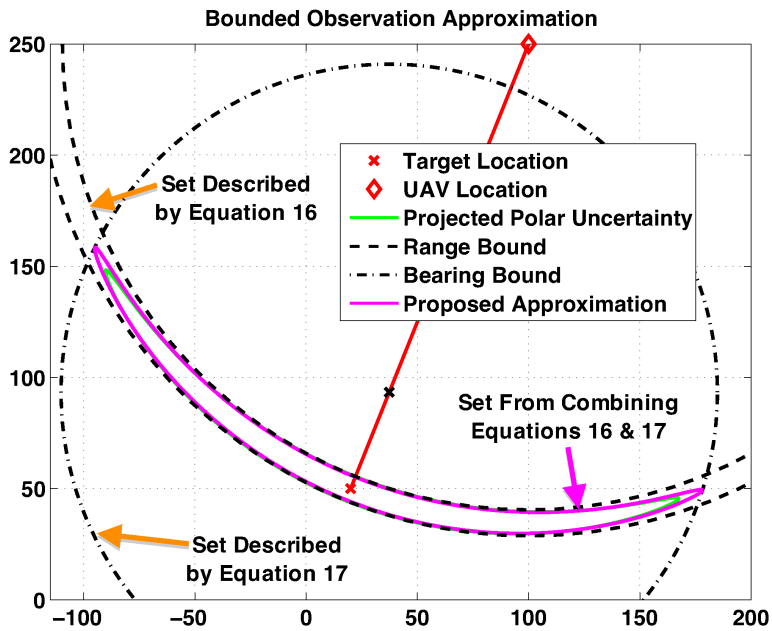

Fig. 6: Tight observation uncertainty approximation constructed in over-parameterized state space

\section{RESUlTS}

\section{A. Field Experiments in Stationary Target Geolocation}

The proposed approach has been tested in real-time on data collected from a number of live flights of a commercial handlaunched UAV over several months of field trials, in varying wind conditions often inducing disturbances exceeding the bandwidth of onboard sensors. Ground objects of interest in the UAV camera's view were designated by an operator and then tracked through succeeding video frames by an adaptive mean-shift tracker [19] and a variation of the Lucas-Kanade algorithm [20]. 
Figure 8 shows a series of such pixel observations over one orbit of a ground target passed through $f_{\text {obs }}(\cdot)$ relative to the true location surveyed by a high-accuracy GPS unit. A timevarying bias is clearly present, and individual observation errors exceed 50 meters. Figure 9 shows a progression of the proposed filter using these observations. Despite the large uncertainty of individual observations, the filtered estimate of the target's location converges rapidly to within a much smaller uncertainty than the tightest 2D ellipse enclosing each observation. This is confirmed numerically by Figure 7, which importantly also shows that the estimate is consistent, the error lying within the predicted uncertainty bounds.

A comparison to alternative estimation methods in terms of order of complexity, the ability to accurately represent observation and posterior estimate sets and the ability to handle moving targets is summarized in Table I. The proposed method is compared to the Cartesian ellipsoidal parametric representations used in Extended and Unscented Kalman filters, and non-parametric methods based on particle sampling and discrete grid representations. The key strength of nonparametric methods is their ability to approximate arbitrary uncertainty. Cartesian parametric filters fail to adequately capture observation and estimate uncertainty while grid based methods are poorly suited to modelling target motion. While not able to express arbitrary uncertainty, the proposed compact representation expresses uncertainty sets appropriate for target tracking by small UAVs. The distinguishing factor is complexity in terms of processing and storage requirements. Our evaluation considers state dimension $d$, dimension of the error vector $e$ and the appropriate number of samples or grid cells $N$. Given $d=2<e \ll N$, the proposed method delivers an appropriate uncertainty approximation with significantly lower computational complexity than non-parametric estimation methods.

TABLE I: Comparison with standard estimation methods

\begin{tabular}{|c|c|c|c|}
\hline Filter & Complexity & Representation & Motion \\
\hline \hline EKF & $d^{3}$ & poor & moderate \\
\hline UKF & $d^{3}+2 e$ & moderate & moderate \\
\hline PF & $N$ & good (arbitrary) & good \\
\hline Grid & $N$ & good (arbitrary) & poor \\
\hline Proposed & $(d+1)^{3}$ & good (appropriate) & moderate \\
\hline
\end{tabular}

\section{CONCLUSION}

We demonstrated a filter for accurate and efficient target geolocation in the presence of large, non-linear, and nonGaussian UAV pose uncertainty. Such uncertainty is a situation typically encountered in small UAV operations with cheap, light-weight and low accuracy sensing.

The proposed geolocation uncertainty representation avoids the challenge associated with attempting to recover accurate UAV pose. We accurately represent the complex nature of the vehicle's state uncertainty. Combining observations using an over-parameterized state representation provides a close approximation to the true uncertainty.

We demonstrate our filter with real data in the field by comparing geolocation results against ground truth surveyed

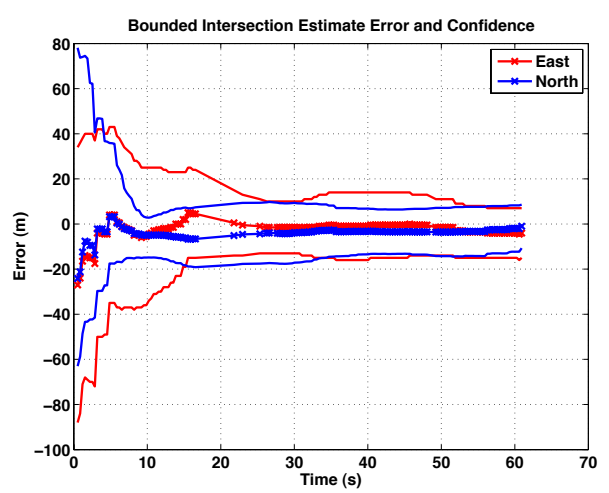

Fig. 7: Stationary target estimate confidence over time, computed from the same flight data as Figures 8 and 9. Crosses connected by the center line are the current mean of the filter. The outer lines represent the confidence bounds.

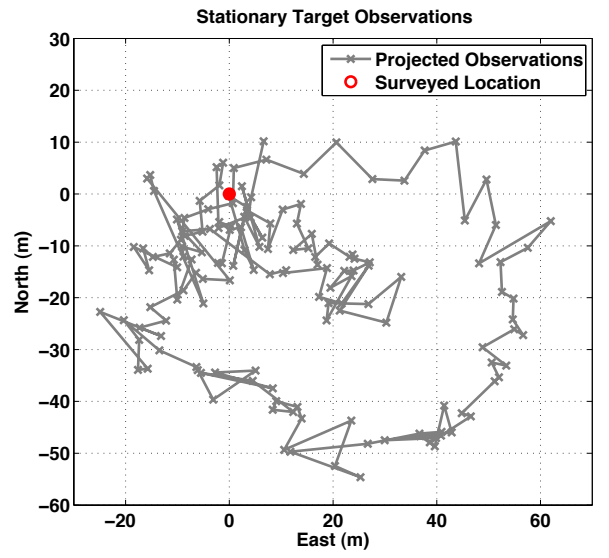

Fig. 8: Stationary target observed locations over time. The grey crosses are projected given the state provided by the vehicles' onboard sensors. There are large errors $(>100 \mathrm{~m})$ in geolocating if no filtering is applied to the observation.

locations. The filter converges to a consistent solution with a tight confidence despite highly uncertain observations.

In future work we aim to prove the filter's applicability to moving targets and leverage this compact representation in distributed collaborative target tracking where efficient communication and computation usage is critical.

\section{ACKNOWLEDGEMENTS}

The field experiments in this work are funded by the US Army ARDEC contract W15QKN-08-C-0497 under subcontract to iRobot Corporation. The authors wish to thank ARDEC and Mark Moseley from iRobot for their support.

\section{REFERENCES}

[1] D. B. Barber, J. D. Redding, T. W. Mclain, R. W. Beard, and C. N. Taylor, "Vision-based target geo-location using a fixed-wing miniature air vehicle," J. Intell. Robotics Syst., vol. 47, no. 4, pp. 361-382, 2006.

[2] D. Gibbins, P. Roberts, and L. Swierkowski, "A video geo-location and image enhancement tool for small unmanned air vehicles (UAVs)," in Intelligent Sensors, Sensor Networks and Information Processing Conference, Dec. 2004, pp. 469-473.

[3] G. Conte, M. Hempel, P. Rudol, D. Lundström, S. Duranti, M. Wzorek, and P. Doherty, "High accuracy ground target geo-location using autonomous micro aerial vehicle platforms," in AIAA Conference on Guidance, Navigation, and Control, August 2008. 

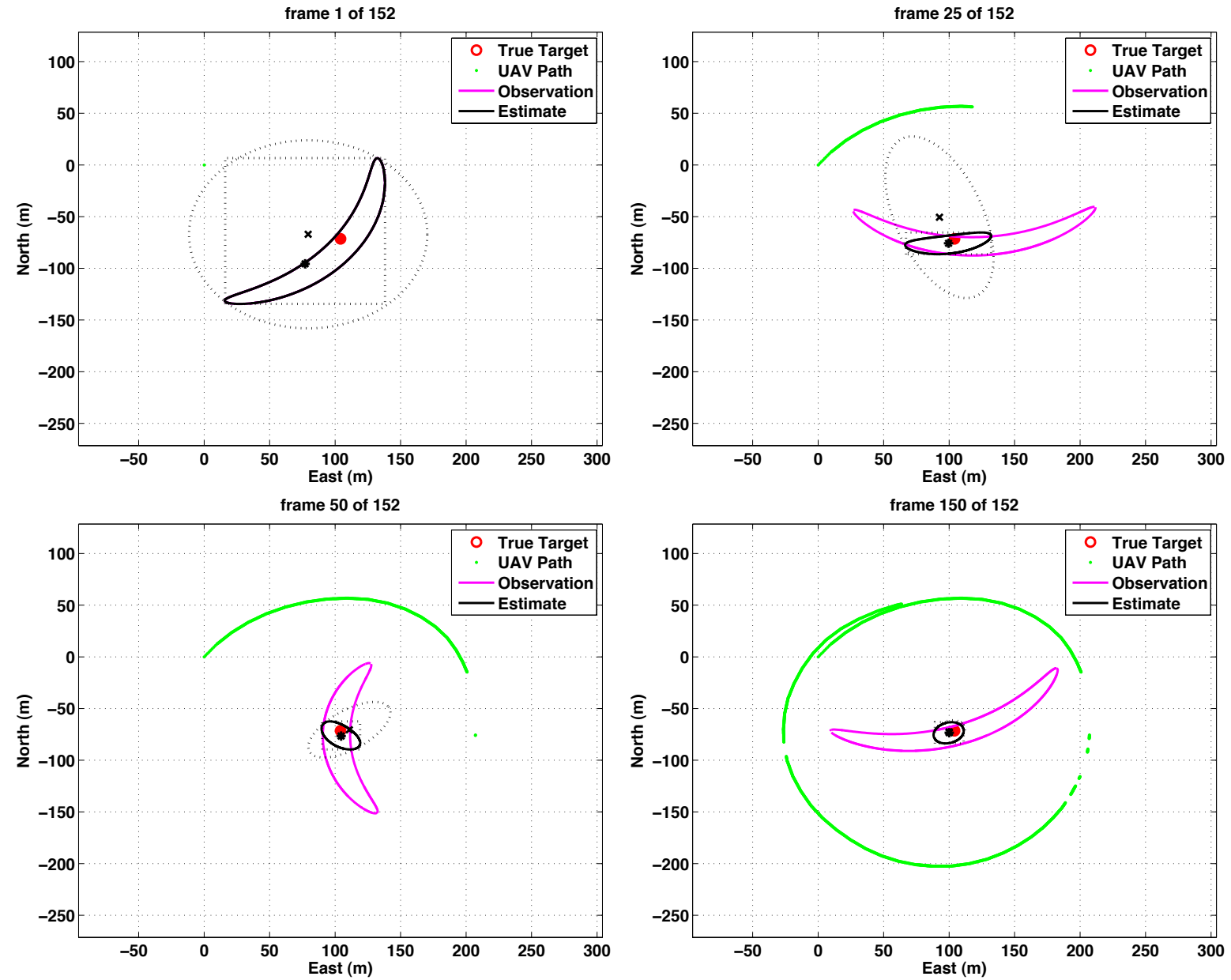

Fig. 9: Snapshots of stationary target location estimate from the same flight data from Figure 8. The UAV path is drawn in green, the surveyed location of the target highlighted by a red circle. The dotted ellipse is a conservative and fast approximation of the proposed filter's confidence based only using the linear portion of the state representation. The dotted box is a tighter approximation requiring slightly more computation. Magenta crescent is the observation level-set and the black crescent is the current estimate level-set taken from the filter. Both the observation and estimate level-set are computed from an explicit analytic polynomial solution.

[4] M. Bryson and S. Sukkarieh, "Bearing-only SLAM for an airborne vehicle," in Australasian Conf. on Robotics and Automation, 2005.

[5] F. Caballero, L. Merino, J. Ferruz, and A. Ollero, "Vision-based odometry and SLAM for medium and high altitude flying UAVs," J. Intell. Robotics Syst., vol. 54, no. 1-3, pp. 137-161, 2009.

[6] R. Madison, P. DeBitetto, A. Rocco Olean, and M. Peebles, "Target geolocation from a small unmanned aircraft system," in IEEE Aerospace Conference, March 2008, pp. 1-19.

[7] E. Andersen and C. Taylor, "Improving MAV pose estimation using visual information," in IEEE International Conference on Intelligent Robots and Systems (IROS), November 2007, pp. 3745-3750.

[8] J. A. Ross, B. R. Geiger, G. L. Sinsley, J. F. Horn, L. N. Long, and A. F. Niessner, "Vision-based target geolocation and optimal surveillance on an unmanned aerial vehicle," in AIAA Conference on Guidance, Navigation, and Control, August 2008.

[9] V. N. Dobrokhodov, I. I. Kaminer, K. D. Jones, and R. Ghabcheloo, "Vision-based tracking and motion estimation for moving targets using small UAVs," in American Control Conference, June 2006.

[10] I. H. Wang, V. N. Dobrokhodov, I. I. Kaminer, and K. D. Jones, "On vision-based target tracking and range estimation for small UAVs," in AIAA Conf. on Guidance, Navigation, and Control, August 2005.

[11] S. J. Julier and J. K. Uhlmann, "A new extension of the Kalman filter to nonlinear systems," in International Symposium on Aerospace/Defense Sensing, Simulation, and Controls, 1997.

[12] M. S. Arulampalam, S. Maskell, and N. Gordon, "A tutorial on particle filters for online nonlinear/non-gaussian bayesian tracking,"
IEEE Transactions on Signal Processing, vol. 50, pp. 174-188, 2002.

[13] M. Quigley, M. Goodrich, S. Griffiths, A. Eldredge, and R. Beard, "Target acquisition, localization, and surveillance using a fixed-wing mini-UAV and gimbaled camera," in IEEE International Conference on Robotics and Automation (ICRA), April 2005, pp. 2600-2605.

[14] J. Djugash, S. Singh, and B. P. Grocholsky, "Modeling mobile robot motion with polar representations," in IEEE International Conference on Intelligent Robots and Systems (IROS), October 2009.

[15] S. Nuske, M. Dille, B. Grocholsky, and S. Singh, "Representing substantial heading uncertainty for accurate target geolocation by small UAVs," in AIAA Conference on Guidance, Navigation, and Control, August 2010.

[16] F. C. Schweppe, "Recursive state estimation: unknown but bounded errors and system inputs," in IEEE Transactions on Automatic Control, vol. AC(13)-1, 1968, pp. 22-28.

[17] K. Briechle and U. Hanebeck, "Localization of a mobile robot using relative bearing measurements," in IEEE Transactions on Robotics and Automation, vol. 20(1), 2004, pp. 36-44.

[18] E. Stump, B. Grocholsky, and V. Kumar, "Extensive representations and algorithms for nonlinear filtering and estimation," in Workshop on Algorithmic Foundations in Robotics (WAFR), 2006.

[19] R. T. Collins and Y. Liu, "On-line selection of discriminative tracking features," in IEEE Conf. on Computer Vision, 2003, pp. 346-352.

[20] S. Baker and I. Matthews, "Lucas-kanade 20 years on: A unifying framework," International Journal of Computer Vision, vol. 56, no. 3, pp. 221-255, 2004. 